\title{
Role of mitofusin 2 in the protective effect of breviscapine against hepatic ischemia/reperfusion injury in rats
}

\author{
ZHEN BAO, WEIJUN CHEN, FAN PAN, BO PENG and JIN GONG \\ Department of General Surgery, The First Affiliated Hospital of Jinan University, Guangzhou, Guangdong 510630, P.R. China
}

Received October 11, 2017; Accepted January 23, 2018

DOI: $10.3892 /$ etm.2018.5834

\begin{abstract}
The purpose of the present study was to investigate the effect of breviscapine injection on hepatic ischemia/reperfusion (I/R) injury in rats. To explore the relevance and discuss the underlying mechanism of mitofusin 2 (Mfn2) in hepatic I/R injury, 40 Sprague-Dawley male rats were randomly and equally divided into five groups $(n=8$ per group) as follows: Sham, I/R + normal saline 1 (NS1), $\mathrm{I} / \mathrm{R}+$ breviscapine 1 (Bre1), I/R + NS2 and I/R + Bre 2 groups. Groups 1 and 2 represented ischemia for 20 and $60 \mathrm{~min}$, respectively. Breviscapine or normal saline was injected via the tail vein (single dose of $10 \mathrm{mg} / \mathrm{kg}$ ) $1 \mathrm{~h}$ prior to surgery and immediately postoperatively. The classical model of hepatic $\mathrm{I} / \mathrm{R}$ injury was used in the present study. The blood and liver samples of different groups were collected following reperfusion to observe serum transaminases and histopathological changes. Alterations in Mfn2, cytosolic cytochrome $c$ and cleaved caspase-3 were additionally assessed. The results demonstrated that breviscapine improved liver function, based on histopathological analysis, and decreased levels of the liver enzymes aspartate and alanine aminotransferase in the $I / R+$ Bre groups compared with the $I / R+N S$ group $(\mathrm{P}<0.05)$. The expression of Mfn2 was significantly increased in the $\mathrm{I} / \mathrm{R}+$ Bre groups $(\mathrm{P}<0.05)$, whereas the expression of caspase-3 and cytosolic cytochrome $c$ protein was decreased in the $\mathrm{I} / \mathrm{R}+$ Bre groups $(\mathrm{P}<0.05)$ compared with the $\mathrm{I} / \mathrm{R}+\mathrm{NS}$ group. These data provided substantial evidence that breviscapine treatment exerted a protective effect against damage induced by hepatic I/R. This protective effect was possibly due to its ability to inhibit I/R-induced apoptosis and promote the expression of Mfn2.
\end{abstract}

Correspondence to: Dr Jin Gong, Department of General Surgery, The First Affiliated Hospital of Jinan University, 613 West of Huangpu Avenue, Guangzhou, Guangdong 510630, P.R. China E-mail: gongjin153@163.com

Key words: hepatic ischemia/reperfusion, breviscapine, apoptosis, mitofusin 2

\section{Introduction}

The occurrence of hepatic ischemia/reperfusion (I/R) injury is inevitable in cases of trauma, shock, hepatectomy and hepatic transplantation. These events may lead to the compromise of liver function and structure, particularly inpatients with hepatic steatosis or cirrhosis. To date, the exact pathogenesis of I/R injury remains unclear. I/R injury involves a series of complex pathophysiological mechanisms, and apoptosis is one of the most important mechanisms of cell death after hepatic I/R injury (1). I/R injury induces apoptosis of hepatic cells and results in the compromise of hepatic function (1). Therefore, it may be inferred that inhibition of apoptosis is a promising approach for the preservation of hepatic function.

The mitochondrial apoptosis pathway is a key step in the process of apoptosis (2), and the release of cytochrome $c$ from mitochondria plays a key role. Under conditions of $\mathrm{I} / \mathrm{R}$ injury, the fine balance between mitochondrial fusion and fission within a cell may be disrupted, as well as mitochondrial homeostasis, predisposing the cell to apoptosis $(3,4)$. This suggests that protection of mitochondria may play an important role in the maintenance of cellular integrity during $\mathrm{I} / \mathrm{R}$ injury (5). Mitofusin 2 (Mfn2), a protein that is present in the outer membrane of the mitochondrion, is a dynamin-like protein that mediates fusion of the mitochondria and plays an important role in the regulation of mitochondrial morphology and function $(6,7)$. The fragmentation of mitochondria is aggravated in apoptotic hepatocytes when the expression of Mfn2 is downregulated in hepatic I/R injury (8). Therefore, investigating changes in the expression of Mfn2 may help to determine the role of the mitochondrial apoptosis pathway in hepatic I/R injury.

Breviscapine, a traditional Chinese medicine, is a flavonoid derived from the natural plant Erigeron breviscapus. Scutellarin is the main active ingredient and its structural formula is 4,5,6-trihydroxyflavone-7-glucuronide (9). Previous studies have demonstrated that breviscapine decreases cardiomyocyte apoptosis and neuroapoptosis during I/R injury $(10,11)$, and breviscapine is known to possess protective propertiesin myocardial and cerebral I/R injury. However, studieson its protective effects against hepatic I/R injury are currently scarce. Our previous study demonstrated that breviscapine preconditioning attenuates hepatic I/R injury by inhibiting the development of liver oxidative stress (12). Therefore, it may be inferred that the 
reduction of hepatocellular apoptosis may be involved in the protective effect of breviscapine against hepatic I/R injury.

Based on these findings, a hepatic $I / R$ rat model was established in the present study to investigate the protective effect of breviscapine against hepatic I/R injury after different durations of ischemia. The results revealed that breviscapine reduced cell apoptosis in hepatic I/R injury through upregulation of the expression of the Mfn2 protein. The aim of the present study was to provide a theoretical basis for the clinical application of breviscapine to treat hepatic I/R injury.

\section{Materials and methods}

Experimental animals and treatment design. A total of 40 male Sprague-Dawley rats, weighing 225-250 g, were purchased from Jinan Peng Yue Experimental Animal Breeding Co., Ltd. (Shandong, China) and maintained in a temperature-controlled room with alternating $12 \mathrm{~h}$ light-dark cycles under pathogen-free conditions. All animal study protocols used in the present study were approved by the Laboratory Animal Ethics Committee of Jinan University and conducted in accordance with their guidelines. The 40 male Sprague-Dawley rats were randomly divided into five groups ( $n=8$ per group) as follows: i) Sham group: The animals underwent midline laparotomy only, without vessel occlusion. ii) I/R + NS1 group: Equivalent volume of normal saline was administered via the tail vein $1 \mathrm{~h}$ prior to surgery and immediately postoperatively. Hepatic reperfusion was recovered after $20 \mathrm{~min}$ of ischemia and the abdomen was closed. iii) I/R + Brel group: A breviscapine injection (10 mg/kg; Hunan Hang Seng Pharmaceutical Co., Ltd., Hong Kong, China) was administered via the tail vein $1 \mathrm{~h}$ prior to surgery and immediately postoperatively. Hepatic reperfusion was recovered after $20 \mathrm{~min}$ of ischemia and the abdomen was closed.iv) I/R + NS2 group: All surgical procedures were performed as in the I/R + NS1 group, but the time of ischemia was increased to $60 \mathrm{~min}$. v) $\mathrm{I} / \mathrm{R}+\mathrm{Bre} 2$ group $(n=8)$ : All surgical procedures were performed as in the I/R + NS2 group, but breviscapine $(10 \mathrm{mg} / \mathrm{kg})$ instead of normal saline was injected via the tail vein. All the rats were fasted but allowed free access to water for $12 \mathrm{~h}$ prior to surgery and were anesthetized with $10 \%$ chloralhydrate $(4 \mathrm{ml} / \mathrm{kg}$, i.p.). Surgery was performed as previously described (13). Briefly, laparotomy was performed through a small midline incision and the hilum of the liver was exposed. The hepatic artery, portal vein and bile duct to the left anterior and median hepatic lobes were clamped using a non-invasive vascular clamp, leading to ischemia of almost $70 \%$ of the liver. The clamps were removed after the aforementioned times. After $6 \mathrm{~h}$ of reperfusion, the animals were sacrificed, and blood and liver tissue samples were collected and immediately stored at $-80^{\circ} \mathrm{C}$.

Serum aspartate and alanine aminotransferase (AST and ALT) levels. In order to determine the degree of hepatic injury, blood was collected in sterile syringes without anticoagulant and then centrifuged to separate the serum. The serum AST and ALT levels were determined using a Selectra-E auto analyzer.

Histological assessment. The liver tissue wasfixed in $10 \%$ neutral buffered formalin and the specimens were embedded in paraffin. The tissue were sliced into $4-\mu \mathrm{m}$ sections and stained with hematoxylin and eosin (H\&E) for histopathological examination. The histological severity of the hepatic injury was evaluated under a light microscope by a pathologist who was blinded to the research. Histological examination was based on the standard procedures reported previously (14).

Tissue apoptosis assayed by TUNEL. Hepatocellular apoptosis was assessed using the terminal deoxynucleotidyl transferase deoxyuridine triphosphate (dUTP) nick end labeling (TUNEL). TUNEL staining was performed according to the manufacturer's instructions using an in situ apoptosis detection kit (Bio-Techne China Co., Ltd., Shanghai, China). Apoptotic cells exhibiteda brown-stained nucleus and were identified among viablecells. The results were expressed as the proportion of TUNEL-positive cells amongthe total number of hepatocytes in 5 non-overlapping serial scopes taken from each slide, with a random start, at x200 magnification.

Western blotting. The levels of cleaved caspase-3, cytoplasm cytochrome $c$ and Mfn2 were determined using western blot analysis. The levels of cytochrome $c$ were determined in cytoplasmic extracts as previously described (15). Briefly, liver tissues were ground and lysed in lysis buffer (Promega Corporation, Madison, WI, USA). The lysates were centrifuged at $850 \mathrm{x} \mathrm{g}$ for $10 \mathrm{~min}$ at $4^{\circ} \mathrm{C}$ to remove the nuclei and cell debris, and the supernatants were further centrifuged at $10,000 \times \mathrm{g}$ for $10 \mathrm{~min}$ at $4^{\circ} \mathrm{C}$. Subsequently, the supernatants were collected for cytoplasmic cytochrome $c$ analysis. Protein concentration was determined by the BCA protein assay (Pierce; Thermo Fisher Scientific, Inc., Waltham, MA, USA). An equal amount of protein from each sample was separated by homogeneous $10 \%$ SDS-polyacrylamide gels and then transferred onto PVDF membranes. The membranes were blocked with 5\% fat-free milk blocking buffer at room temperature and then incubated with primary antibodies overnight at $4^{\circ} \mathrm{C}$. After being washed with TBST buffer, the corresponding secondary antibodies were used to identify primary antibody binding. After washing, the bands were visualized using enhanced chemiluminescence reagents (ECL; Bio-Rad Laboratories, Inc., Hercules, CA, USA) and the FluorChem 5500 imaging system (Alpha Innotech Corp., San Leandro, CA, USA). The band intensities were evaluated by densitometric analysis using Image $\mathrm{J} 1.50$ software (National Institutes of Health, Bethesda, MD, USA).

Reverse transcription-quantitative polymerase chain reaction $(R T-q P C R)$. Total RNA was extracted from hepatic tissues using TRIzol reagent (Invitrogen; Thermo Fisher Scientific, Inc.) according to the manufacturer's protocols. The RNA purity and concentration were determined spectrophotometrically using the NanoDrop ND-1000 (NanoDrop Technologies; Thermo Fisher Scientific, Inc., Wilmington, DE, USA). RNA (100 ng) was reverse-transcribed into complementary DNA (cDNA) with PrimeScript RT Master Mix (Takara Biotechnology Co., Ltd., Dalian, China) in a $20 \mathrm{ml}$ final reaction volume, according to the manufacturer's protocol. The primer sequences (5'-3') were as follows: $\beta$-actin (forward) TGCTATGTTGCCCTAGAC TTCG, (reverse) GTTGGCATAGAGGTCTTTACGG; Mfn2 (forward) GATGACAGAGGAAGTGGAAAGGC, (reverse) ACAGACACAGGAAGAAGGGGCT. Relative expression 
A

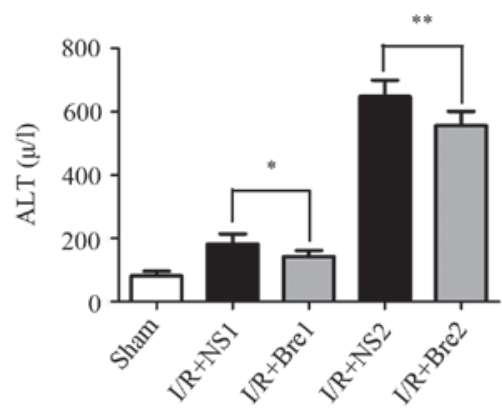

B

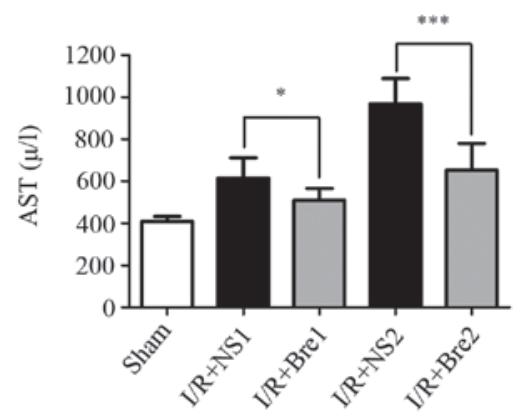

Figure 1. Breviscapine treatment reduced serum transaminase level following ischemia/reperfusion (I/R) injury. Serum samples were collected $6 \mathrm{~h}$ after $\mathrm{I} / \mathrm{R}$ injury for measuring (A) serum alanine aminotransferase (ALT) and (B) serum aspartate aminotransferase (AST). Data are expressed as the mean \pm standard deviation $(\mathrm{n}=8) .{ }^{*} \mathrm{P}<0.05,{ }^{* *} \mathrm{P}<0.01,{ }^{* * *} \mathrm{P}<0.001$ vs. normal saline (NS) groups.

A

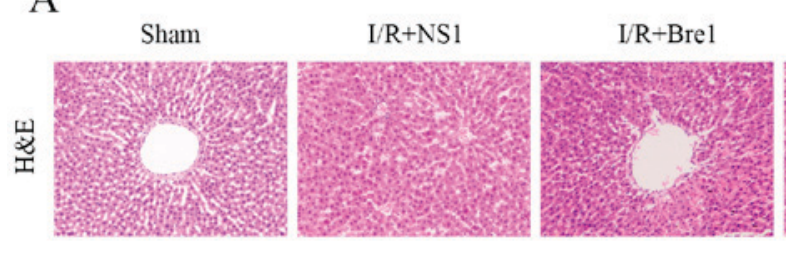

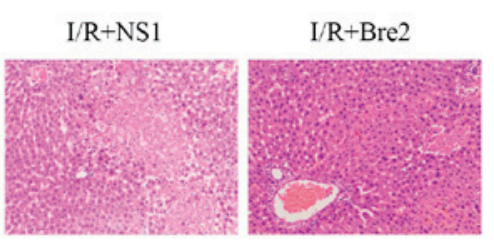

$\mathrm{B}$

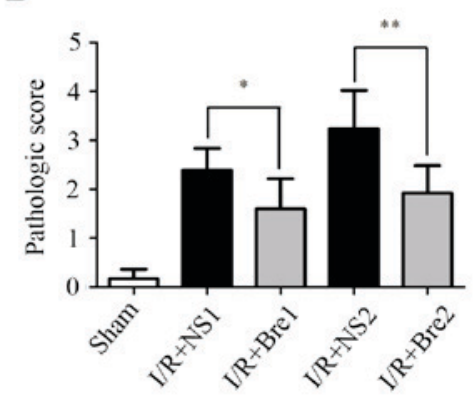

Figure 2. Breviscapine treatment markedly attenuated ischemia/reperfusion (I/R) injury-induced pathological changes. (A) Histological findings of the liver tissue in the sham, I/R + NS1, I/R + Bre1, I/R + NS2 and I/R + Bre2 groups. Photomicrographs of the liver tissue stained with hematoxylin and eosin (original magnification, x100). (B) Pathological score following hepatic I/R injury in rats. Data are expressed as the mean \pm standard deviation $(\mathrm{n}=8)$. ${ }^{*} \mathrm{P}<0.05,{ }^{* * *} \mathrm{P}<0.01$ vs. NS groups. Bre, breviscapine; NS, normal saline.

was measured by qPCR using SYBR Green PCR Master Mix SYBR Premix Ex Taq ${ }^{\mathrm{TM}}$ II (Takara Biotechnology Co., Ltd.,) on a Mastercycler ep realplex4 (Eppendorf, Hamburg, Germany). Mfn2 gene expression profiles were normalized to $\beta$-actin and calculated using real-time quantitative PCR and the $2^{-\Delta \Delta C q}$ method.

Statistical analysis. Data analysis was performed using the SPSS statistical package, version 13 (SPSS, Inc., Chicago, IL, USA). The results are expressed as the mean \pm standard deviation and one-way analysis of variance was used to compare groups. $\mathrm{P}<0.05$ was considered to indicate a statistically significant difference.

\section{Results}

Breviscapine alleviates I/R-induced deterioration of hepatic function. The serum AST and ALT levels in each group are shown in Fig. 1. The levels of serum ALT and AST in the sham group were $81.63 \pm 14.49$ and $409.8 \pm 23.06 \mathrm{U} / 1$, respectively. After 20 and $60 \mathrm{~min}$ of ischemia and $6 \mathrm{~h}$ of reperfusion, the levels of serum ALT and AST were significantly increased in the two groups compared with the sham group $(\mathrm{P}<0.01)$. The serum levels of ALT and AST were significantly decreased in the $\mathrm{I} / \mathrm{R}+$ Bre groups compared with those in the $\mathrm{I} / \mathrm{R}+\mathrm{NS}$ groups $(\mathrm{P}<0.05)$, particularly in the $\mathrm{I} / \mathrm{R}+\mathrm{Bre} 2$ group $(\mathrm{P}<0.01)$. These results indicated that breviscapine can reduce the serum level of ALT and AST compared with normal saline. These data were associated with histological tissue alterations and H\&E staining revealed that the structure of hepatic tissue in the sham group appeared normal (Fig. 2A). Compared with the sham group, the livers of rats in the normal saline control groups exhibited extensive hepatocyte swelling with significantly more prominent vacuolar degeneration, unclear boundaries, narrower hepatic sinusoids and hepatocellular necrosis, accompanied by a large number of infiltrating neutrophils, particularly in the I/R + NS2 group. Tissues from the $\mathrm{I} / \mathrm{R}+$ Bre groups exhibited less prominent neutrophil 
A
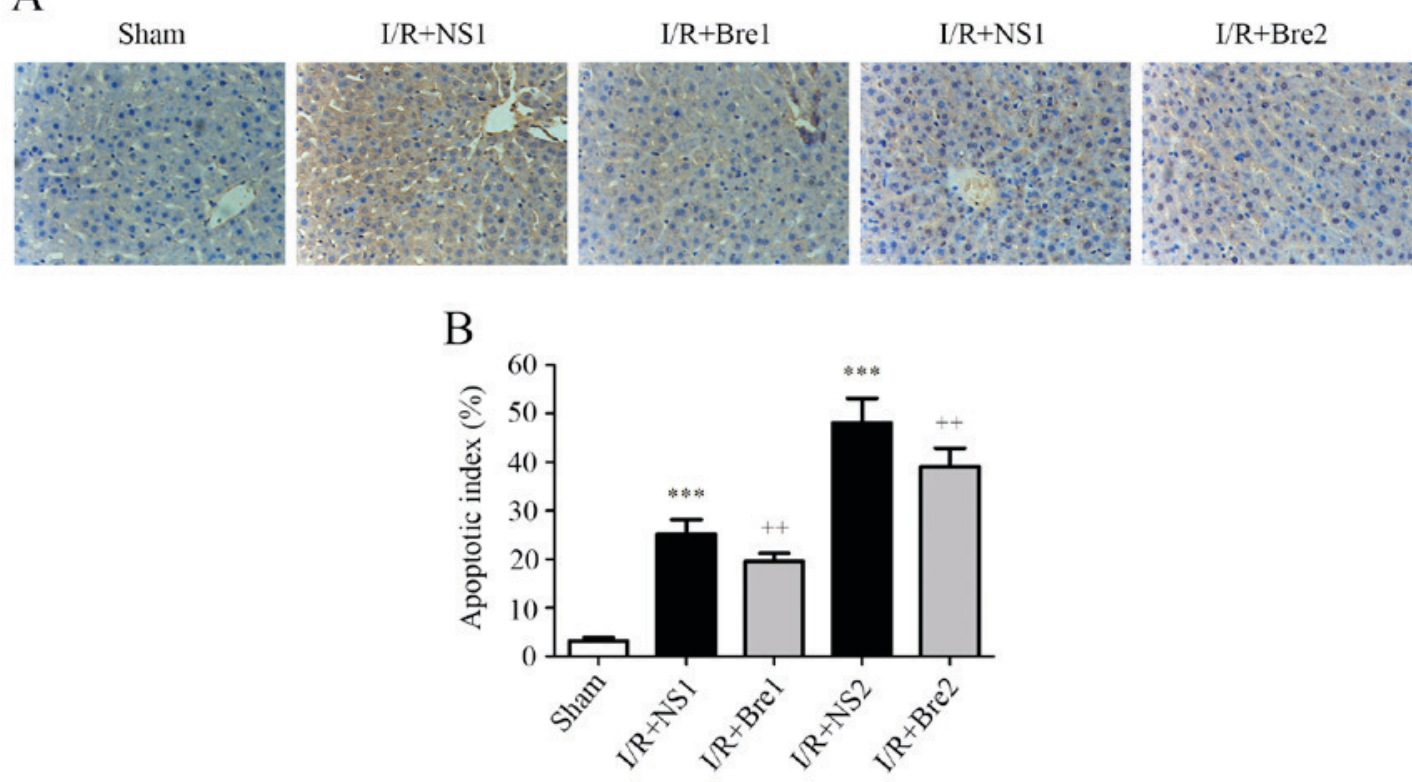

Figure 3. Breviscapine treatment markedly reduced ischemia/reperfusion (I/R) injury-induced apoptosis. (A) Cell apoptosis was analyzed using TUNEL staining (original magnification, x200). (B) The apoptotic index (AI) is quantified as the ratio of TUNEL-positive cells to total number of hepatocytes. Data are expressed as the mean \pm standard deviation $(\mathrm{n}=8) .{ }^{* * *} \mathrm{P}<0.001$ vs. Sham group; ${ }^{++} \mathrm{P}<0.01$ vs. normal saline (NS) groups.

infiltration, necrosis and hepatocyte swelling, particularly in the I/R + Bre2 group. As shown in Fig. 2B, tissues from the normal saline control groups exhibited a higher pathological score compared with the sham group. The level of hepatic function injury improved in the I/R + Bre groups, particularly in the $\mathrm{I} / \mathrm{R}+\mathrm{Bre} 2$ group $(\mathrm{P}<0.01)$ when compared with the normal saline control groups. These results demonstrated that breviscapine improved the compromised hepatic function induced by $I / R$, and this protective effect became more pronounced with prolongation of the time of ischemia.

Breviscapine reduces the apoptotic index (AI) of hepatocytes following $I / R$ injury. A TUNEL assay was used to assess the level of apoptosis and AI was used to assess the protective effect of breviscapine. As shown in Fig. 3, the AI observably increased in the normal saline groups compared with the sham group $(\mathrm{P}<0.001)$. Furthermore, the AI in the $\mathrm{I} / \mathrm{R}+\mathrm{Bre} 1$ and $\mathrm{I} / \mathrm{R}+\mathrm{Bre} 2$ groups was markedly reduced compared with the normal saline groups $(\mathrm{P}<0.01)$. These results suggest that breviscapine protected the hepatocytes against I/R-induced apoptosis.

Breviscapine restores elevated cleaved caspase-3 expression in the hepatic and cytosolic translocation of cytochrome $c$. To further explore the possible mechanism by which breviscapine reduces hepatocyte apoptosis, the expression level of caspase-3 and cytochrome $c$ was detected by western blotting. As shown in Fig. 4, the normal saline groups exhibited a higher expression of cytochrome $c$ and cleaved caspase-3 compared with the sham group $(\mathrm{P}<0.05)$, and this discrepancy became more evident with prolongation of the time of ischemia, particularly in the $\mathrm{I} / \mathrm{R}+\mathrm{NS} 2$ group $(\mathrm{P}<0.01)$. These results demonstrated that breviscapine reduced the release of mitochondrial cytochrome $c$ and the expression of cleaved caspase- 3 compared with the normal saline groups $(\mathrm{P}<0.05)$. It is well established that the release of cytochrome $c$ is associated with caspase family activation. Our findings suggest that breviscapine plays a role in preventing mitochondrial-related hepatocyte apoptosis by suppressing cytochrome $c$ release and caspase activation during I/R injury.

Breviscapine downregulates the expression of $M f n 2$ in hepatocytes during I/R injury. Mfn2 is a mediator during mitochondrial fusion, an evolutionarily conserved process responsible for the surveillance of mitochondrial homeostasis. To further explore the effect of breviscapine on mitochondrial function, changes in the expression of Mfn2 were detected by western blotting. The results demonstrated that the expression of Mfn2 in the I/R + NS groups was significantly lower compared with the sham group $(\mathrm{P}<0.05)$. The expression of Mfn2 in the I/R + Bre groups was significantly higher compared with the I/R + NS groups $(\mathrm{P}<0.05$; Fig. 5). Finally, RT-qPCR was performed to further confirm the hypothesis that the mRNA concentration of Mfn2 in the normal saline groups wassignificantly decreased compared with that in the sham group $(\mathrm{P}<0.05)$. However, the mRNA concentrations of Mfn2 in the I/R + Bre groups were increased compared with those in the normal saline groups (Fig. 5). Our findings indicate that breviscapine protects hepatocytes against I/R injury by upregulating the expression of Mfn2.

\section{Discussion}

With the growing number of patients undergoing liver transplantation or hepatectomy, hepatic I/R injury is inevitable; this type of injury may also occur in other clinical settings, including trauma and hemorrhagic shock. Hepatic I/R injury not only results in liver dysfunction, but also increases mortality rate and the period of hospitalization. Therefore, alleviating the effects of hepatic I/R injury is urgent in clinical practice. 
A

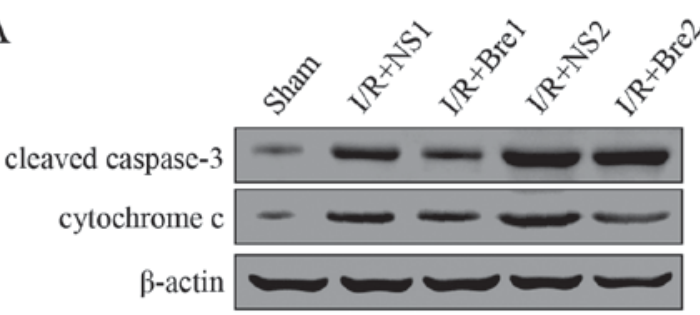

B

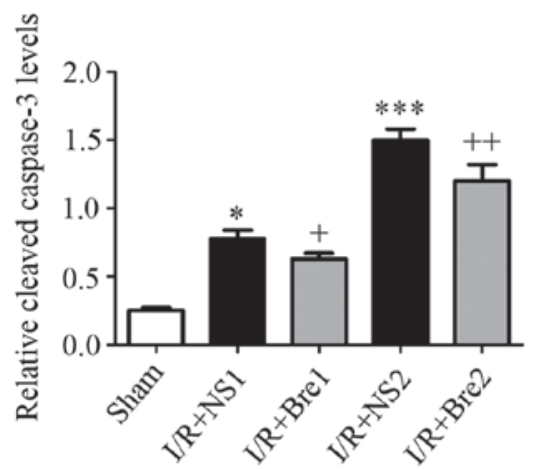

$\mathrm{C}$

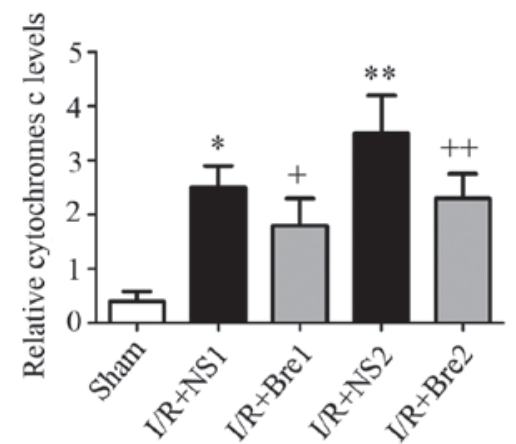

Figure 4. Breviscapine treatment limited the increase in cleaved caspase-3 expression in the hepatic and cytosolic translocation of cytochrome $c$. (A) The protein levels of cleaved caspase-3 and cytochrome $c$ were determined via western blot analysis. (B) The bar chart demonstrates the relative expression of cleaved caspase-3. (C) The bar chart demonstrates the relative expression of cleaved cytochrome $c$. Data are expressed as the mean \pm standard deviation. ${ }^{*} \mathrm{P}<0.05,{ }^{* *} \mathrm{P}<0.01,{ }^{* * * *} \mathrm{P}<0.001$ vs. sham group; ${ }^{+} \mathrm{P}<0.05,{ }^{++} \mathrm{P}<0.01$ vs. normal saline (NS) groups.

A

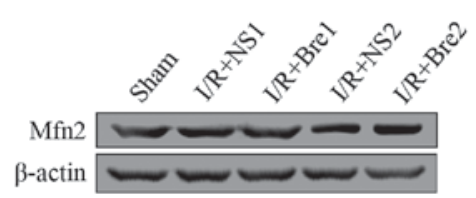

B

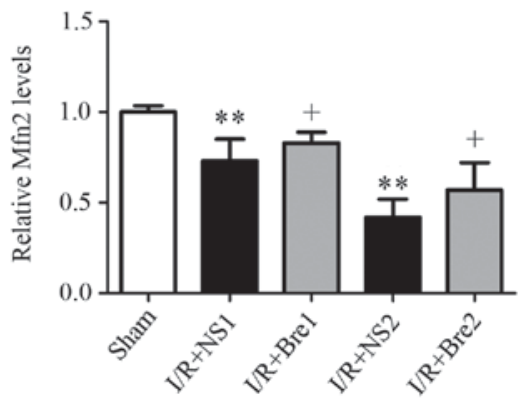

$\mathrm{C}$

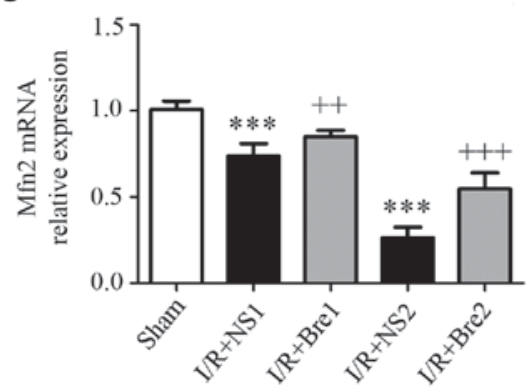

Figure 5. Breviscapine treatment limited the downregulation of Mfn2 expression in hepatocytes following ischemia/reperfusion (I/R) injury. (A) The protein levels of Mfn2 were determined via western blot analysis. (B) The bar chart demonstrates the relative expression of Mfn2. (C) The mRNA levels of Mfn2 were determined via reverse transcription-polymerase chain reaction analysis. Data are expressed as the mean \pm standard deviation. ${ }^{* *} \mathrm{P}<0.01,{ }^{* * *} \mathrm{P}<0.001 \mathrm{vs}$, sham group; ${ }^{+} \mathrm{P}<0.05,{ }^{++} \mathrm{P}<0.01,{ }^{++} \mathrm{P}<0.001$ vs. NS groups. Mfn2, mitofusin 2 ; NS, normal saline.

Breviscapine is a widely used traditional Chinese medicine that is derived from the natural plant Erigeron breviscapus. It is extensively used in clinical settings for treating cerebral infarction, cardiovascular disease and stroke in China. It was reported that breviscapine decreased cardiomyocyte apoptosis and neuroapoptosis during I/R injury $(10,11)$. However, whether breviscapine is able to suppress hepatic I/R injury remains obscure. In the present study, breviscapine was found to decrease ALT and AST levels, ameliorate histological findings and inhibit subsequent hepatocyte apoptosis following hepatic I/R injury in rats. Therefore, it was hypothesized that breviscapine protects hepatocytes from I/R-induced injury 
and the mitochondrial-dependent apoptotic pathway may be involved in its protective effect.

The serum levels of ALT and AST reflect the degree of liver function damage. Any injury to the cellular membrane of hepatocytes, such as I/R-induced hepatic injury, may result in a rapid increase of the serum ALT level (16). If the hepatic injury persists, AST expression in the mitochondria and circulation would be increased (17). The present study demonstrated that liver tissues from the breviscapine-treated groups exhibited a smaller increase in ALT and AST levels compared with the normal saline-treated groups. The protective effect of breviscapine was also supported by the fact that the pathomorphological changes of the liver tissue in the $\mathrm{I} / \mathrm{R}+$ Bre groups were milder compared with those in the normal saline groups. These results suggest that breviscapine exerted a protective effect on hepatocytes and reduced I/R injury.

Apoptosis is the major type of cell death and it plays a crucial role in hepatic I/R injury (1). Mitochondria play a key role in the regulation of cell apoptosis. It was previously demonstrated that I/R injury leads to the opening of a non-specific pore in the inner mitochondrial membrane, referred to as the mitochondrial permeability transition pore (mPTP) (18). The opening of the mPTP results in dissipation of the mitochondrial membrane potential $(\Delta \Psi \mathrm{m})$, followed by progressive mitochondrial swelling and the loss of soluble components of the respiratory chain, which eventually leads to rupture of the outer mitochondrial membrane and leakage of the pro-apoptotic protein cytochrome $c$ from the mitochondria to the cytosol (19). I/R-induced mPTP opening leads to $\Delta \Psi \mathrm{m}$ collapse and cytochrome $c$ release from mitochondria to the cytosol, where it interacts with the apoptosis protease-activating factor-1 (APAF-1) that causes activation of caspase-9, which in turn activates caspase-3 $(20,21)$. Caspase-3 is a major factor in the process of cell apoptosis and its activated form, cleaved caspase-3, marks the irreversible phase of cell apoptosis.

We herein hypothesized that breviscapine may be able to reduce apoptosis in hepatic I/R injury via reducing the activity of mitochondria-related apoptotic pathways. In the present study, it was observed that breviscapine effectively downregulated the expression of cleaved caspase- 3 in hepatocytes and reduced the translocation of cytochrome $c$ from mitochondria to the cytosol. The results suggested that breviscapine may exert anti-apoptotic effects against hepatic I/R injury.

Mitochondria are dynamic organelles and their morphological transitions are mainly effected by undergoing fission and fusion, processes essential to mitochondrial homeostasis $(21,22)$. The fine balance between mitochondrial fusion and fission within a cell may be affected by I/R injury (3), which promotes mPTP opening (23) and predisposes the cell to apoptosis (4). Mfn2, a mitochondrial outer membrane protein, is essential for mitochondrial fusion and it regulates mitochondrial metabolism and cell death (6). The fragmentation of mitochondria is aggravated when the expression of Mfn2 is suppressed, and it observably increases the sensitivity of the cell to apoptotic signals (24). Mfn2 is vital for maintaining the mitochondrial structure. It has been demonstrated that Mfn2 not only preserves mitochondrial morphology in hepatocytes, but also is associated with the opening of mPTP in the liver under conditions of I/R injury (25). Ong et al (22) also reported that the overexpression of $\mathrm{Mfn} 2$ prevented
mPTP opening induced by I/R injury in the HL-1 cardiac cell line. As mentioned above, mPTP opening leads to cytochrome $c$ release from the mitochondria to the cytosol. It has been reported that upregulating the expression of Mfn2 may reduce the release of cytochrome $c$ (26), while downregulating the expression of Mfn2 exacerbates ceramide-induced mitochondrial dysfunction and release of cytochrome $c$ (27). These findings indicate that Mfn2 reduces apoptosis through inhibiting the mitochondrial apoptosis pathway. In the present study, the expression of Mfn2 was found to be significantly decreased in the normal saline groups compared with that in the sham group, while the level of Mfn2 in the I/R + Bre groups increased compared with that in the normal saline groups. These results suggest that the protective effect of breviscapine against hepatic I/R maybe effected via upregulating the expression of Mfn2.

In summary, our results demonstrated the protective effect of breviscapine against hepatic I/R injury in an animal model. The mechanism underlying this protective effect is likely through inhibiting the mitochondrial apoptosis pathway and upregulating Mfn2 expression, which reduces hepatocyte apoptosis. Therefore, breviscapine may have potential as a novel therapeutic agent for the treatment of hepatic I/R injury and may be of value in a clinical setting. However, further studies are required to fully elucidate the mechanisms underlying its effects.

\section{Acknowledgements}

The present study was supported by the Science and Technology Program of Guangzhou, China (grant no. 2011j4100078).

\section{References}

1. Kong R, Gao Y, Sun B, Chen H, Wang G, Wang X, Zhu H, Pan S, Xue D and Jiang H: The strategy of combined ischemia preconditioning and salvianolic acid-B pretreatment to prevent hepatic ischemia-reperfusion injury in rats. Dig Dis Sci 54: 2568-2576, 2009.

2. Green DR and Kroemer G: The pathophysiology of mitochondrial cell death. Science 305: 626-629, 2004.

3. Brady NR, Hamacher-Brady A and Gottlieb RA: Proapoptotic BCL-2 family members and mitochondrial dysfunction during ischemia/reperfusion injury, a study employing cardiac HL-1 cells and GFP biosensors. Biochim Biophys Acta 1757: 667-678, 2006.

4. Frank S, Gaume B, Bergmann-Leitner ES, Leitner WW, Robert EG, Catez F, Smith CL and Youle RJ: The role of dynamin-related protein 1, a mediator of mitochondrial fission, in apoptosis. Dev Cell 1: 515-525, 2001.

5. Novgorodov SA and Gudz TI: Ceramide and mitochondria in ischemia/reperfusion. J Cardiovasc Pharmacol 53: 198-208, 2009.

6. Zorzano A, Liesa M, Sebastián D, Segalés J and Palacín M: Mitochondrial fusion proteins: Dual regulators of morphology and metabolism. Semin Cell Dev Biol 21: 566-574, 2010.

7. Huang P, Galloway C and Yoon Y: Control of mitochondrial morphology through differential interactions of mitochondrial fusion and fission proteins. PLoS One 6: e20655, 2011.

8. Li J, Ke W, Zhou Q, Wu Y, Luo H, Zhou H, Yang B, Guo Y, Zheng Q and Zhang Y: Tumour necrosis factor- $\alpha$ promotes liver ischaemia-reperfusion injury through the PGC-1 $\alpha / \mathrm{Mfn} 2$ pathway. J Cell Mol Med 18: 1863-1873, 2014.

9. Lou XY, Cheng JL and Zhang B: Therapeutic effect and mechanism of breviscapine on cisplatin-induced nephrotoxicity in mice. Asian Pac J Trop Med 8: 873-877, 2015.

10. Wang J, Ji SY, Liu SZ, Jing R and Lou WJ: Cardioprotective effect of breviscapine: Inhibition of apoptosis in H9c2 cardiomyocytes via the PI3K/Akt/eNOS pathway following simulated ischemia/reperfusion injury. Pharmazie 70: 593-597, 2015. 
11. Yiming L, Wei H, Aihua L and Fandian Z: Neuroprotective effects of breviscapine against apoptosis induced by transient focal cerebral ischaemia in rats. J Pharm Pharmacol 60: 349-355, 2008.

12. Lin YZ, Lu ZY, Liang XH, Li K, Peng B and Gong J: Effect of breviscapine against hepatic ischemia reperfusion injury. J Surg Res 203: 268-274, 2016.

13. Nauta RJ, Tsimoyiannis E, Uribe M, Walsh DB, Miller D and Butterfield A: Oxygen-derived free radicals in hepatic ischemia and reperfusion injury in the rat. Surg Gynecol Obstet 171: 120-125, 1990.

14. Wei Y, Chen P, de Bruyn M, Zhang W, Bremer E and Helfrich W: Carbon monoxide-releasing molecule-2 (CORM-2) attenuates acute hepatic ischemia reperfusion injury in rats. BMC Gastroenterol 10: 42, 2010.

15. Gomez L, Paillard M, Thibault H, Derumeaux G and Ovize M: Inhibition of GSK3beta by postconditioning is required to prevent opening of the mitochondrial permeability transition pore during reperfusion. Circulation 117: 2761-2768, 2008.

16. Li H, Sun JJ, Chen GY, Wang WW, Xie ZT, Tang GF and Wei SD: Carnosic acid nanoparticles suppress liver ischemia/reperfusion injury by inhibition of ROS, Caspases and NF- $\kappa \mathrm{B}$ signaling pathway in mice. Biomed Pharmacother 82 : 237-246, 2016.

17. Kamiike W, Fujikawa M, Koseki M, Sumimura J, Miyata M Kawashima Y, Wada H and Tagawa K: Different patterns of leakage of cytosolic and mitochondrial enzymes. Clin Chim Acta 185: 265-270, 1989.

18. Halestrap AP, Clarke SJ and Javadov SA: Mitochondrial permeability transition pore opening during myocardial reperfusion-a target for cardioprotection. Cardiovasc Res 61: 372-385, 2004.

19. Baines CP, Kaiser RA, Purcell NH, Blair NS, Osinska H, Hambleton MA, Brunskill EW, Sayen MR, Gottlieb RA, Dorn GW, et al: Loss of cyclophilin D reveals a critical role for mitochondrial permeability transition in cell death. Nature 434: $658-662,2005$
20. Hüttemann M, Helling S, Sanderson TH, Sinkler C, Samavati L, Mahapatra G, Varughese A, Lu G, Liu J, Ramzan R, et al: Regulation of mitochondrial respiration and apoptosis through cell signaling: Cytochrome $\mathrm{c}$ oxidase and cytochrome $\mathrm{c}$ in ischemia/reperfusion injury and inflammation. Biochim Biophys Acta 1817: 598-609, 2012.

21. Nakagawa T, Shimizu S, Watanabe T, Yamaguchi O, Otsu K, Yamagata $\mathrm{H}$, Inohara H, Kubo $\mathrm{T}$ and Tsujimoto $\mathrm{Y}$ : Cyclophilin D-dependent mitochondrial permeability transition regulates some necrotic but not apoptotic cell death. Nature 434: 652-658, 2005.

22. Ong SB, Subrayan S, Lim SY, Yellon DM, Davidson SM and Hausenloy DJ: Inhibiting mitochondrial fission protects the heart against ischemia/reperfusion injury. Circulation 121: 2012-2022, 2010.

23. Kong D, Xu L, Yu Y, Zhu W, Andrews DW, Yoon Y and Kuo TH: Regulation of $\mathrm{Ca} 2+-$ induced permeability transition by Bcl-2 is antagonized by Drpl and hFis1. Mol Cell Biochem 272: 187-199, 2005.

24. Liu X, Feng L, Yan M, Xu K, Yu Y and Zheng X: Changes in mitochondrial dynamics during amyloid $\beta$-induced PC12 cell apoptosis. Mol Cell Biochem 344: 277-284, 2010.

25. Biel TG, Lee S, Flores-Toro JA, Dean JW, Go KL, Lee MH, Law BK, Law ME, Dunn WA Jr, Zendejas I, et al: Sirtuin 1 suppresses mitochondrial dysfunction of ischemic mouse livers in a mitofusin 2-dependent manner. Cell Death Differ 23: 279-290, 2016

26. Neuspiel M, Zunino R, Gangaraju S, Rippstein $P$ and McBride H: Activated mitofusin 2 signals mitochondrial fusion, interferes with Bax activation, and reduces susceptibility to radical induced depolarization. J Biol Chem 280: 25060-25070, 2005.

27. Papanicolaou KN, Khairallah RJ, Ngoh GA, Chikando A, Luptak I, O'Shea KM, Riley DD, Lugus JJ, Colucci WS, Lederer WJ, et al: Mitofusin-2 maintains mitochondrial structure and contributes to stress-induced permeability transition in cardiac myocytes. Mol Cell Biol 31: 1309-1328, 2011. 